\title{
IFNL4 ss469415590 Variant Is Associated with Treatment Response in Japanese HCV Genotype 1 Infected Individuals Treated with IFN-Including Regimens
}

\author{
Tatsuo Miyamura, ${ }^{1}$ Tatsuo Kanda, ${ }^{1}$ Shingo Nakamoto, ${ }^{2}$ Makoto Arai, ${ }^{1}$ \\ Masato Nakamura, ${ }^{1}$ Shuang Wu, ${ }^{1}$ Xia Jiang, ${ }^{1}$ Reina Sasaki, ${ }^{1}$ Yuki Haga, \\ Shin Yasui, ${ }^{1}$ Yoshihiko Ooka, ${ }^{1}$ Tetsuhiro Chiba, ${ }^{1}$ Fumio Imazeki, \\ Shigeru Mikami, ${ }^{3}$ and Osamu Yokosuka ${ }^{1}$ \\ ${ }^{1}$ Departments of Gastroenterology and Nephrology, Graduate School of Medicine, Chiba University, \\ 1-8-1 Inohana, Chuo-ku, Chiba 260-8677, Japan \\ ${ }^{2}$ Department of Molecular Virology, Graduate School of Medicine, Chiba University, Chiba 260-8677, Japan \\ ${ }^{3}$ Department of Gastroenterology, Kikkoman General Hospital, Noda 278-0005, Japan \\ Correspondence should be addressed to Tatsuo Kanda; kandat-cib@umin.ac.jp
}

Received 17 July 2014; Accepted 24 November 2014; Published 8 December 2014

Academic Editor: Shigeru Marubashi

Copyright ( $(2014$ Tatsuo Miyamura et al. This is an open access article distributed under the Creative Commons Attribution License, which permits unrestricted use, distribution, and reproduction in any medium, provided the original work is properly cited.

\begin{abstract}
Aim. Eradication of hepatitis C virus (HCV) is still challenging even if interferon- (IFN-) free regimens with direct-acting antiviral agents (DAAs) for HCV-infected individuals are available in clinical practice. IFNL4 is a newly described protein, associated with human antiviral defenses. We investigated whether IFNL4 ss469415590 variant has an effect on the prediction of treatment response in HCV-infected patients treated with IFN-including regimens. Patients and Methods. In all, 185 patients infected with HCV genotype 1 treated with peg-IFN plus ribavirin, with or without telaprevir, were genotyped for IFNL4 ss469415590. We retrospectively investigated whether the role of IFNL4 ss469415590 variant and other factors could predict sustained virological response (SVR) in Japanese patients infected with HCV genotype 1. Results. There were $65.7 \%$, 31.5\%, and 2.8\% patients in the IFNL4 ss469415590 TT/TT, TT/-G, and -G/-G groups, respectively. SVR rates were $82.1 \%$ or $49.3 \%$ in patients treated with pegIFN plus ribavirin with or without telaprevir, respectively. IFNL4 ss469415590 variant and HCV viral loads or IFNL4 ss469415590 variant and early virological response were better predictors of SVR in patients treated with peg-IFN plus ribavirin with or without telaprevir, respectively. Conclusion. In the era of DAAs, measurement of IFNL4 ss469415590 variant could help the prediction of SVR in Japanese HCV genotype 1 infected individuals treated with IFN-including regimens.
\end{abstract}

\section{Introduction}

Chronic hepatitis C virus (HCV) infection is a leading cause of hepatocellular carcinoma (HCC) and end-stage liver disease requiring liver transplantation in the United States $[1,2]$. In Japan, HCV infection is still a major cause of HCC [3]. At present, $\mathrm{HCV}$ infection is a world health problem.

The former standard treatment with peginterferon (pegIFN) plus ribavirin led to $\sim 50 \%$ and $\sim 80 \%$ sustained virological response (SVR) rates in patients infected with HCV genotypes 1 and 2, respectively [4]. Since 2011, peg-IFN plus ribavirin with telaprevir or boceprevir has been available in the United States as well as that with telaprevir in Japan. These treatments resulted in higher SVR rates $(\sim 70 \%$ and $\sim 80 \%$ for treatment-naïve patients and previous treatment relapsers, resp.) in $\mathrm{HCV}$ genotype 1 infected patients [5].

Genome-wide association studies (GWAS) [6-8] revealed that several single nucleotide polymorphisms (SNPs) near the IFNL3 (previously called IL28B) coding region, such as rs8099917 and rs12978690, are associated with treatment 
response to peg-IFN plus ribavirin treatment. We also reported that IFNL3 rs8099917 was useful for the prediction of SVR in patients infected with HCV genotype 1 [9]. However, the mechanism between IFNL3 and clearance of HCV has been unclear despite the extensive work [10-12]. We also reported that IFNL3 induces IFN-stimulated genes (ISGs) such as IFN- $\gamma$-inducible protein (IP-10) that are reportedly associated with the progression of $\mathrm{HCV}$-related pathogenesis and antiviral activities against HCV [12].

A recent study [13] showed that a variant upstream of IFNL3 could create IFNL4, impairing the clearance of HCV. Bibert et al. [14] reported that induction of IL28B and IP-10 mRNA relies on IFNL4 ss469415590 TT/-G variant in peripheral blood mononuclear cells (PBMCs), and this variant would improve the prediction of treatment response to peg-IFN and ribavirin $[14,15]$.

So far, only a few studies are available about the association between IFNL4 ss469415590 variant and treatment response in Japanese patients infected with HCV genotype 1. Peg-IFN plus ribavirin with telaprevir is also still one of the standard care treatments for chronic hepatitis $\mathrm{C}$ genotype 1 patients, at least until IFN-free regimens become generally available in clinical daily practice.

In the present study, the usefulness of IFNL4 ss469415590 variant for the prediction of virological response in chronic hepatitis $\mathrm{C}$ patients treated with peg-IFN plus ribavirin with or without telaprevir was investigated.

\section{Patients and Methods}

2.1. Patients. The present study comprised a total of 185 patients with chronic hepatitis $C$ treated with peg-IFN plus ribavirin with or without telaprevir at the Department of Gastroenterology, Chiba University Hospital, and the Department of Gastroenterology, Kikkoman General Hospital in Chiba, Japan. Of these patients, 81 patients treated with pegIFN plus ribavirin had been already included in previous reports $[9,11,16]$. Written informed consent was obtained from all patients, and the study was approved by the Ethics Committee of Chiba University, School of Medicine (number 428). All patients were of Asian origin and infected with $\mathrm{HCV}$ genotype 1 . They underwent standard antiviral therapy with the combination of peg-IFN plus ribavirin [4] or telaprevir with peg-IFN plus ribavirin $[17,18]$. HCV RNA was measured at baseline, at weeks 4 and 12, at the end of treatment, and at 24 weeks after the end of treatment. SVR was defined as undetectable serum HCV RNA at 24 weeks after the end of treatment. Patients with undetectable HCV RNA at 4 and 12 weeks after the commencement of treatment were considered to have had rapid virological response (RVR) and early virological response (EVR), respectively [19]. Relapse was defined as undetectable HCV RNA at the end of treatment, but it reappeared after the end of treatment [20]. Null response was defined as less than $2 \log _{10}$ decrease in HCV RNA (IU/mL) from baseline after 12 weeks of therapy [20].

2.2. DNA Extraction and IFNL4 Variant Genotyping. Blood DNA was extracted by DNA extracted all lysis reagents
(Applied Biosystems, Foster City, CA, USA). IFNL4 ss469415590 was determined by TaqMan SNP assay. Primers were purchased from Applied Biosystems. Thermal cycling was performed with the ABI Step One real-time PCR system as previously described [9]. We analyzed IFNL4 ss469415590 TT/TT as major genotype and TT/-G and -G/-G as minor genotypes in the present study.

2.3. Measurement of HCV RNA in Serum. HCV RNA was measured by COBAS AMPLICOR HCV Monitor test v. 2.0 (range: $0.5-850 \mathrm{KIU} / \mathrm{mL}$, lower limit of detection: $1.7 \mathrm{log}$ $\mathrm{IU} / \mathrm{mL}$ ) or COBAS TaqMan HCV test with levels ranging from 1.2 to $7.8 \log \mathrm{IU} / \mathrm{mL}$ (Roche Diagnostics, Tokyo, Japan). In all patients treated with telaprevir with peg-IFN plus ribavirin, HCV RNA was measured by COBAS TaqMan HCV test.

2.4. Statistical Analysis. Data are expressed as mean \pm SD. Variables with categorical data were compared among groups using chi-square test and Student's $t$-test as indicated. $P<$ 0.05 was considered statistically significant. Variables with $P<0.1$ at univariate analysis were retained for multivariate logistic regression analysis. Statistical analysis was performed using the Excel statistics program for Windows, version 7 (SSRI, Tokyo, Japan).

\section{Results}

IFNL4 ss469415590 variant was determined in 178 of the total 185 patients, and 117 (65.7\%) had TT/TT, 56 (31.5\%) had TT/G, and 5 (2.8\%) had -G/-G of ss469415590 in the present study (Table 1). In 141 patients treated with peg-IFN plus ribavirin without telaprevir, 93 (66.0\%) had TT/TT, 43 (30.5\%) had TT/-G, and 5 (3.5\%) had -G/-G of ss469415590 (Table 2). In 37 patients treated with peg-IFN, ribavirin, and telaprevir, 24 (64.9\%) had TT/TT and 13 (35.1\%) had TT/-G of ss469415590 (Table 3). The distribution of IFNL4 ss469415590 variant of both treatment groups was quite similar.

In 24 -week follow-up after treatment, $82.1 \%$ or $49.3 \%$ of patients treated with peg-IFN plus ribavirin with or without telaprevir, respectively, achieved SVR (Table 1). To clarify the predictors of SVR, we compared the baseline characteristics and treatment response between SVR and non-SVR groups of the patients treated with peg-IFN plus ribavirin (Table 4(a)). Univariate analysis showed that previous treatment response, $\gamma$-GTP levels, and platelet count, having RVR, EVR, and IFNL4 ss469415590 variant in the patients treated with pegIFN plus ribavirin, contributed to the achievement of SVR. Factors associated with SVR by univariate analysis were then subjected to multivariate logistic regression analysis. SVR was attained independently of IFNL4 ss469415590 major genotype and having EVR in these patients treated with pegIFN plus ribavirin (Table 4(b)).

Next, we compared the baseline characteristics and treatment response between SVR and non-SVR groups of the patients treated with peg-IFN, ribavirin, and telaprevir (Table 5(a)). HCV RNA at baseline was included, as the measurement of HCV RNA detection was uniform in this group 
TABLE 1: Baseline characteristics of patients in the present study.

\begin{tabular}{|c|c|c|c|c|}
\hline & $\begin{array}{c}\text { All patients, } \\
n=185\end{array}$ & $\begin{array}{l}\text { Patients with dual } \\
\text { therapy, } n=146\end{array}$ & $\begin{array}{l}\text { Patients with triple } \\
\text { therapy, } n=39\end{array}$ & $\begin{array}{c}P \text { values (dual therapy } \\
\text { versus triple therapy } \\
\text { groups) }\end{array}$ \\
\hline Gender, male/female & $102 / 83$ & $73 / 73$ & $29 / 10$ & 0.011 \\
\hline Age, years & $56.2 \pm 10.7$ & $55.7 \pm 11.2$ & $58.2 \pm 8.7$ & 0.197 \\
\hline $\begin{array}{l}\text { Previous treatment: } \\
\text { naïve/relapse/null }\end{array}$ & $119 / 45 / 21$ & $102 / 26 / 18$ & $17 / 19 / 3$ & $\begin{array}{c}0.0043 \\
\text { (naïve versus non-naïve) }\end{array}$ \\
\hline AST, IU/L & $57.1 \pm 48.0$ & $58.0 \pm 48.7$ & $53.9 \pm 45.9$ & 0.630 \\
\hline ALT, IU/L & $70.4 \pm 64.7$ & $71.0 \pm 64.9$ & $68.1 \pm 64.8$ & 0.800 \\
\hline$\gamma$-GTP, IU/L & $57.0 \pm 66.7$ & $56.4 \pm 68.6$ & $59.2 \pm 59.8$ & 0.816 \\
\hline Hemoglobin, g/dL & $14.1 \pm 1.3$ & $14.0 \pm 1.2$ & $14.6 \pm 1.3$ & 0.0070 \\
\hline Platelets, $\times 10^{4} / \mathrm{mm}^{3}$ & $16.6 \pm 5.6$ & $16.8 \pm 5.8$ & $16.0 \pm 5.1$ & 0.434 \\
\hline \multicolumn{5}{|l|}{ Treatment response } \\
\hline $\mathrm{RVR}, n$ & 42 & 15 & 27 & $<0.0001$ \\
\hline EVR, $n$ & 99 & 65 & 34 & $<0.0001$ \\
\hline SVR/relapse/null & $104 / 39 / 42$ & $72 / 37 / 37$ & $32 / 2 / 5$ & $<0.0001$ \\
\hline $\begin{array}{l}\text { IFNL4, ss } 469415590 \text {, } \\
\text { major/minor/ND }\end{array}$ & $117 / 61 / 7$ & $93 / 48 / 5$ & $24 / 13 / 2$ & 0.944 \\
\hline
\end{tabular}

Peginterferon plus ribavirin with or without telaprevir therapy was defined as triple or dual therapy, respectively. Null: null response; AST: aspirate aminotransferase; ALT: alanine aminotransferase; $\gamma$-GTP: $\gamma$-glutamyltransferase; RVR: rapid virological response; EVR: early virological response; SVR: sustained virological response.

TABLE 2: Baseline characteristics and treatment response in 141 patients treated with peginterferon plus ribavirin according to IFNL4 ss469415590 variant.

\begin{tabular}{lccc}
\hline & IFNL4 major, $n=93$ & IFNL4 minor, $n=48$ & $P$ values (major versus minor groups) \\
\hline Gender, male/female & $46 / 47$ & $25 / 23$ & 0.900 \\
Age, years & $56.1 \pm 11.7$ & $54.7 \pm 10.6$ & 0.488 \\
Previous treatment: naïve/relapse/null & $68 / 19 / 6$ & $29 / 7 / 12$ & 0.0042 \\
AST, IU/L & $53.4 \pm 48.5$ & $65.0 \pm 44.0$ & 0.167 \\
ALT, IU/L & $65.1 \pm 64.9$ & $82.0 \pm 63.3$ & 0.14 \\
$\gamma$-GTP, IU/L & $40.9 \pm 39.2$ & $87.5 \pm 98.9$ & 0.000107 \\
Hemoglobin, g/dL & $13.9 \pm 1.2$ & $14.2 \pm 1.2$ & 0.16 \\
Platelets, $\times 10^{4} / \mathrm{mm}^{3}$ & $16.8 \pm 5.7$ & $16.8 \pm 6.1$ & 1.00 \\
Treatment response & & & 0.0079 \\
RVR, $n$ & 15 & 0 & $<0.0001$ \\
EVR, $n$ & 59 & 5 & $<0.0001$ \\
SVR/relapse/null & $60 / 23 / 10$ & $10 / 12 / 25$ & \\
\hline
\end{tabular}

Null: null response; AST: aspirate aminotransferase; ALT: alanine aminotransferase; $\gamma$-GTP: $\gamma$-glutamyltransferase; RVR: rapid virological response; EVR: early virological response; SVR: sustained virological response.

(see Section 2). Univariate analysis showed that having RVR and EVR in the patients treated with peg-IFN, ribavirin, and telaprevir contributed to achievement of SVR. Factors associated with SVR by univariate analysis were then subjected to multivariate logistic regression analysis. SVR was attained independently of lower HCV RNA at baseline, and IFNL4 ss469415590 major genotype in these patients was treated with peg-IFN plus ribavirin and telaprevir (Table 5(b)).

\section{Discussion}

The present study confirmed previous studies that IFNL4 ss469415590 variant plays an important role in eradicating
HCV genotype 1 from patients treated with peg-IFN plus ribavirin with or without telaprevir [14, 21-23]. Multivariate analysis revealed that IFNL4 ss469415590 major genotype was statistically associated with achieving SVR in patients treated with peg-IFN plus ribavirin as well as those treated with peg-IFN, ribavirin, and telaprevir.

IFNL3-associated SNPs were strongly associated with treatment response to peg-IFN plus ribavirin without telaprevir treatment [6-9]. Although there have been several reports on the mechanism involving IFNL3 $[10,11]$, the exact mechanism or function of IFNL3 has yet to be clarified. Bibert et al. [14] reported that induction of IL28B and IP-10 mRNA relied on ss469415590 TT/-G but not rs12979860, making 
TABLE 3: Baseline characteristics and treatment response in 37 patients treated with peginterferon, ribavirin, and telaprevir according to IFNL4 ss469415590 variant.

\begin{tabular}{lccc}
\hline & IFNL4 major, $n=24$ & IFNL4 minor, $n=13$ & $P$ values (major versus minor groups) \\
\hline Gender, male/female & $19 / 5$ & $9 / 4$ & 0.78 \\
Age, years & $59.5 \pm 7.7$ & $55.2 \pm 10.2$ & 0.15 \\
Previous treatment: naïve/relapse/null & $10 / 13 / 1$ & $7 / 4 / 2$ & 0.71 \\
AST, IU/L & $50.9 \pm 48.9$ & $59.8 \pm 44.2$ & 0.58 \\
ALT, IU/L & $63.8 \pm 60.5$ & $77.9 \pm 77.2$ & 0.54 \\
$\gamma$-GTP, IU/L & $51.8 \pm 52.2$ & $78.6 \pm 73.3$ & 0.25 \\
Hemoglobin, g/dL & $14.6 \pm 1.2$ & $14.5 \pm 1.3$ & 0.81 \\
Platelets, $\times 10^{4} / \mathrm{mm}^{3}$ & $15.9 \pm 5.2$ & $16.5 \pm 5.3$ & 0.74 \\
HCV RNA $(\log$ IU/mL) & $6.70 \pm 0.54$ & $6.18 \pm 0.87$ & 0.0309 \\
Treatment response & & & \\
RVR, $n$ & 17 & 8 & 0.83 \\
EVR, $n$ & 22 & 10 & 0.45 \\
SVR/relapse/null & 22 & 8 & 0.0727 \\
\hline
\end{tabular}

Null: null response; AST: aspirate aminotransferase; ALT: alanine aminotransferase; $\gamma$-GTP: $\gamma$-glutamyltransferase; RVR: rapid virological response; EVR: early virological response; SVR: sustained virological response.

TABLE 4: Comparison of SVR and non-SVR patients treated with peginterferon and ribavirin by univariate analysis (a) and multivariate analysis (b).

(a) Univariate analysis

\begin{tabular}{lccc}
\hline & SVR, $n=72$ & Non-SVR, $n=74$ & $P$ values (SVR versus non-SVR groups) \\
\hline Gender, male/female & $39 / 33$ & $34 / 40$ & 0.40 \\
Age, years & $54.4 \pm 12.7$ & $57.0 \pm 9.3$ & 0.15 \\
Previous treatment: naïve/relapse/null & $59 / 12 / 1$ & $43 / 14 / 17$ & 0.00020 \\
AST, IU/L & $54.1 \pm 54.3$ & $61.8 \pm 42.5$ & 0.34 \\
ALT, IU/L & $69.0 \pm 71.5$ & $73.0 \pm 58.2$ & 0.71 \\
$\gamma$-GTP, IU/L & $42.7 \pm 41.3$ & $70.0 \pm 85.7$ & 0.0158 \\
Hemoglobin, g/dL & $13.9 \pm 1.1$ & $14.1 \pm 1.3$ & 0.31 \\
Platelets, $\times 10^{4} / \mathrm{mm}^{3}$ & $17.8 \pm 5.4$ & $15.7 \pm 5.9$ & 0.0265 \\
Treatment response & & & 0.000107 \\
$\quad$ RVR, $n$ & 15 & 0 & $<0.0001$ \\
EVR, $n$ & 53 & 12 & $<0.0001$ \\
IFNL4, ss469415590, major/minor/ND & $60 / 10 / 2$ & $33 / 38 / 3$ & \\
\hline
\end{tabular}

Null: null response; AST: aspirate aminotransferase; ALT: alanine aminotransferase; $\gamma$-GTP: $\gamma$-glutamyltransferase; RVR: rapid virological response; EVR: early virological response; SVR: sustained virological response.

(b) Factors associated with SVR among patients treated with peginterferon plus ribavirin by multivariate analysis

\begin{tabular}{lcccc}
\hline Factor & Category & Odds ratio & $95 \%$ CI & $P$ values \\
\hline IFNL4, ss469415590 & major/minor & 2.56 & $1.005-6.557$ & 0.0486 \\
EVR & $+/-$ & 10.16 & $4.28-24.10$ & $<0.0001$ \\
\hline
\end{tabular}

EVR: early virological response.

ss469415590 TT/-G the only functional variant identified, although there are contrary opinions $[24,25]$.

Prokunina-Olsson et al. [13] found that several novel transcripts and ss469415590 are specific for the region upstream of IFNL3. Their global protein BLAST search [13] showed that 179 amino acids (p179) from transcripts with the ss469415590-G allele have only homology with $29.1 \%$ amino acid identity and $40.8 \%$ amino acid similarity with IFNL3 among predictive proteins from the 10 novel transcripts.
IFNL3 and these p179 (IFNL4) proteins are most related within the sequences, corresponding to the $\mathrm{A}$ and $\mathrm{F}$ helices of IFNL3, which constitute the core area for interaction of IFNL3 with its primary receptor, IFNLR1 (IL28R1) [13]. The beneficial insertion ss469415590-TT allele could abrogate IFNL4. IFNL4 signaling via IFN $\lambda$ receptor could regulate antiviral activity against coronaviruses as well as HCV [26]. Manuel et al. [27] also reported that polymorphisms in the IFNL3/4 region influence susceptibility to CMV replication 
TABLE 5: Comparison of SVR and non-SVR patients treated with peginterferon, ribavirin, and telaprevir by univariate analysis (a) and multivariate analysis (b).

(a) Univariate analysis

\begin{tabular}{lccc}
\hline & SVR, $n=32$ & Non-SVR, $n=7$ & $P$ values (SVR versus non-SVR groups) \\
\hline Gender, male/female & $24 / 8$ & $5 / 2$ & 0.77 \\
Age, years & $57.8 \pm 9.3$ & $59.8 \pm 5.6$ & 0.58 \\
Previous treatment: naïve/relapse/null & $14 / 17 / 1$ & $3 / 2 / 2$ & 0.13 \\
AST, IU/L & $55.9 \pm 50.1$ & $44.7 \pm 16.0$ & 0.56 \\
ALT, IU/L & $71.9 \pm 70.6$ & $51.1 \pm 19.9$ & 0.44 \\
$\gamma$-GTP, IU/L & $62.1 \pm 64.7$ & $46.2 \pm 27.9$ & 0.53 \\
Hemoglobin, g/dL & $14.6 \pm 1.3$ & $14.4 \pm 1.6$ & 0.72 \\
Platelets, $\times 10^{4} / \mathrm{mm}^{3}$ & $16.4 \pm 5.2$ & $14.3 \pm 4.6$ & 0.33 \\
HCV RNA $(\log$ IU/mL) & $6.45 \pm 0.68$ & $6.81 \pm 0.75$ & 0.220 \\
Treatment response & & & $<0.0001$ \\
$\quad$ RVR, $n$ & 27 & 0 & $<0.0001$ \\
EVR, $n$ & 32 & 2 & 0.0727 \\
IFNL4, major/minor/ND & $22 / 8 / 2$ & $2 / 5 / 0$ & \\
\hline
\end{tabular}

Null: null response; AST: aspirate aminotransferase; ALT: alanine aminotransferase; $\gamma$-GTP: $\gamma$-glutamyltransferase; RVR: rapid virological response; EVR: early virological response; SVR: sustained virological response.

(b) Factors associated with SVR among patients treated with peginterferon, ribavirin, and telaprevir by multivariate analysis

\begin{tabular}{lcccc}
\hline Factor & Category & Odds ratio & $95 \%$ CI & $P$ values \\
\hline HCV RNA $(\log \mathrm{IU} / \mathrm{mL})$ & $<6.4 / 6.4 \leq$ & 13.74 & $1.014-186.30$ & 0.048 \\
IFNL4 & major/minor & 17.54 & $1.980-156.25$ & 0.010 \\
ss469415590 & & & \\
\hline
\end{tabular}

in solid-organ transplant recipients, suggesting that IFNL4 as well as IFNL3 play important roles in human antiviral defenses.

Our results indicated that IFNL4 ss469415590 variant is useful for prediction of the treatment response to IFNincluding regimens. A recent study [28] showed that IFNL4 ss469415590 minor genotype was associated with slower early viral decay, due to slower loss of free virus and decreased drug efficacy in patients treated with the DAA sofosbuvir against HCV NS5B, along with RBV, suggesting that IFNL4 variant might be useful in the DAA era. In IFN-free DAA therapies, it is unclear whether these variants play a role or not, although it is possible that certain DAAs could have effects on IFN signaling such as ISGs of host cells.

\section{Conclusions}

Measurement of IFNL4 ss469415590 variant is another option for prediction of the treatment response to IFN-including regimens in Japanese HCV genotype 1 infected individuals. Together, the mechanism of the interaction between treatment response and IFNL4 as well as IFNL3 in HCV-infected patients should be further explored.

\section{Conflict of Interests}

Tatsuo Kanda reports receiving lecture fees from Chugai Pharmaceutical, MSD, Tanabe-Mitsubishi, Ajinomoto, Bristol-Myers Squibb, Daiichi-Sankyo, Janssen Pharmaceutical, and GlaxoSmithKline. Makoto Arai reports receiving lecture fees from Chugai Pharmaceutical, Eisai, AstraZeneca, Daiichi Sankyo, and Tsumura. Osamu Yokosuka reports receiving grant support from Chugai Pharmaceutical, Bayer, MSD, Daiichi-Sankyo, Tanabe-Mitsubishi, Bristol-Myers Squibb, and Taiho Pharmaceutical. The other authors declare that there is no conflict of interests regarding the publication of this paper.

\section{Authors' Contribution}

Tatsuo Miyamura, Tatsuo Kanda, and Shingo Nakamoto contributed equally to the paper.

\section{Acknowledgments}

The authors thank all their colleagues at the liver units of their hospitals who cared for the patients described herein. This work was supported by Research Grants for Scientific Research from the Ministry of Education, Culture, Sports, Science, and Technology, Japan.

\section{References}

[1] A. M. Di Bisceglie, "Hepatitis C and hepatocellular carcinoma," Hepatology, vol. 26, no. 3, supplement 1, pp. 34S-38S, 1997.

[2] J. W. Ward, "The epidemiology of chronic hepatitis C and onetime hepatitis C virus testing of persons born during 1945 to 
1965 in the United States," Clinics in Liver Disease, vol. 17, no. 1, pp. 1-11, 2013.

[3] I. Saito, T. Miyamura, A. Ohbayashi et al., "Hepatitis C virus infection is associated with the development of hepatocellular carcinoma," Proceedings of the National Academy of Sciences of the United States of America, vol. 87, no. 17, pp. 6547-6549, 1990.

[4] T. Kanda, F. Imazeki, and O. Yokosuka, "New antiviral therapies for chronic hepatitis C," Hepatology International, vol. 4, no. 3, pp. 548-561, 2010.

[5] T. Kanda, O. Yokosuka, and M. Omata, "Treatment of hepatitis $\mathrm{C}$ virus infection in the future," Clinical and Translational Medicine, vol. 2, article 9, 2013.

[6] D. Ge, J. Fellay, A. J. Thompson et al., "Genetic variation in IL28B predicts hepatitis C treatment-induced viral clearance," Nature, vol. 461, no. 7262, pp. 399-401, 2009.

[7] V. Suppiah, M. Moldovan, G. Ahlenstiel et al., "IL28B is associated with response to chronic hepatitis $\mathrm{C}$ interferon- $\alpha$ and ribavirin therapy," Nature Genetics, vol. 41, no. 10, pp. 1100-1104, 2009.

[8] Y. Tanaka, N. Nishida, M. Sugiyama et al., "Genome-wide association of IL28B with response to pegylated interferon- $\alpha$ and ribavirin therapy for chronic hepatitis C," Nature Genetics, vol. 41, no. 10, pp. 1105-1109, 2009.

[9] T. Miyamura, T. Kanda, S. Nakamoto et al., "Roles of ITPA and IL28B genotypes in chronic hepatitis C patients treated with peginterferon plus ribavirin," Viruses, vol. 4, no. 8, pp. 12641278, 2012.

[10] M. Sugiyama, Y. Tanaka, T. Wakita, M. Nakanishi, and M. Mizokami, "Genetic variation of the $I L-28 B$ promoter affecting gene expression," PLoS ONE, vol. 6, no. 10, Article ID e26620, 2011.

[11] T. Miyamura, T. Kanda, S. Nakamoto et al., "Hepatic STAT1nuclear translocation and interleukin $28 \mathrm{~B}$ polymorphisms predict treatment outcomes in hepatitis $\mathrm{C}$ virus genotype 1-infected patients," PLoS ONE, vol. 6, no. 12, Article ID e28617, 2011.

[12] T. Kanda, X. Jiang, S. Nakamoto et al., "Different effects of three interferons $\mathrm{L}$ on toll-like receptor-related gene expression in HepG2 cells," Cytokine, vol. 64, no. 2, pp. 577-583, 2013.

[13] L. Prokunina-Olsson, B. Muchmore, W. Tang et al., "A variant upstream of IFNL3 (IL28B) creating a new interferon gene IFNL4 is associated with impaired clearance of hepatitis C virus," Nature Genetics, vol. 45, no. 2, pp. 164-171, 2013.

[14] S. Bibert, T. Roger, T. Calandra et al., "IL28B expression depends on a novel TT/-G polymorphism which improves HCV clearance prediction," Journal of Experimental Medicine, vol. 210, no. 6, pp. 1109-1116, 2013.

[15] Y. Nozawa, T. Umemura, Y. Katsuyama et al., "Genetic polymorphism in IFNL4 and response to pegylated interferon- $\alpha$ and ribavirin in Japanese chronic hepatitis C patients," Tissue Antigens, vol. 83, no. 1, pp. 45-48, 2014.

[16] S. Nakamoto, T. Kanda, F. Imazeki et al., "Simple assay based on restriction fragment length polymorphism associated with IL28B in chronic hepatitis C patients," Scandinavian Journal of Gastroenterology, vol. 46, no. 7-8, pp. 955-961, 2011.

[17] H. Kumada, J. Toyota, T. Okanoue, K. Chayama, H. Tsubouchi, and N. Hayashi, "Telaprevir with peginterferon and ribavirin for treatment-naive patients chronically infected with HCV of genotype 1 in Japan," Journal of Hepatology, vol. 56, no. 1, pp. 78-84, 2012.

[18] T. Hara, N. Akuta, F. Suzuki et al., "A pilot study of triple therapy with telaprevir, peginterferon and ribavirin for elderly patients with genotype 1 chronic hepatitis C," Journal of Medical Virology, vol. 85, no. 10, pp. 1746-1753, 2013.

[19] T. Kanda, F. Imazeki, Y. Yonemitsu et al., "Quantification of hepatitis $C$ virus in patients treated with peginterferon-alfa 2a plus ribavirin treatment by COBAS TaqMan HCV test," Journal of Viral Hepatitis, vol. 18, no. 7, pp. e292-e297, 2011.

[20] M. Omata, T. Kanda, M.-L. Yu et al., "APASL consensus statements and management algorithms for hepatitis $\mathrm{C}$ virus infection," Hepatology International, vol. 6, no. 2, pp. 409-435, 2012.

[21] Y. Nozawa, T. Umemura, Y. Katsuyama et al., "Genetic polymorphism in IFNL4 and response to pegylated interferon- $\alpha$ and ribavirin in Japanese chronic hepatitis C patients," Tissue Antigens, vol. 83, no. 1, pp. 45-48, 2014.

[22] H. Fujino, M. Imamura, Y. Nagaoki et al., "Predictive value of the IFNL4 polymorphism on outcome of telaprevir, peginterferon, and ribavirin therapy for older patients with genotype $1 \mathrm{~b}$ chronic hepatitis C," Journal of Gastroenterology, 2013.

[23] Y. Nagaoki, M. Imamura, Y. Kawakami et al., "Interferon lambda 4 polymorphism affects on outcome of telaprevir, pegylated interferon and ribavirin combination therapy for chronic hepatitis C," Hepatology Research, 2014.

[24] A. F. Stättermayer, R. Strassl, A. Maieron et al., "Polymorphisms of interferon- $\lambda 4$ and IL28B-effects on treatment response to interferon/ribavirin in patients with chronic hepatitis C," Alimentary Pharmacology and Therapeutics, vol. 39, no. 1, pp. 104111, 2014.

[25] L. M. Real, K. Neukam, R. Herrero et al., "IFNL4 ss469415590 variant shows similar performance to rs 12979860 as predictor of response to treatment against hepatitis $\mathrm{C}$ virus genotype 1 or 4 in Caucasians," PLoS ONE, vol. 9, no. 4, Article ID e95515, 2014.

[26] O. J. Hamming, E. Terczyńska-Dyla, G. Vieyres et al., "Interferon lambda 4 signals via the IFN $\lambda$ receptor to regulate antiviral activity against HCV and coronaviruses," EMBO Journal, vol. 32, no. 23, pp. 3055-3065, 2013.

[27] O. Manuel, A. Wójtowicz, S. Bibert et al., "Influence of IFNL3/4 polymorphisms on the incidence of cytomegalovirus infection after solid-organ transplantation," The Journal of Infectious Diseases, 2014.

[28] E. G. Meissner, D. Bon, L. Prokunina-Olsson et al., "IFNL4- $\Delta$ G genotype is associated with slower viral clearance in Hepatitis $\mathrm{C}$, genotype-1 patients treated with sofosbuvir and ribavirin," Journal of Infectious Diseases, vol. 209, no. 11, pp. 1700-1704, 2014. 


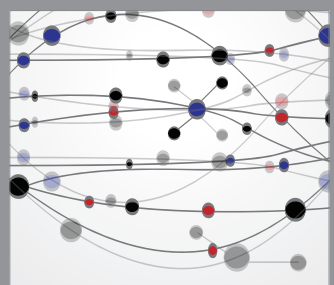

The Scientific World Journal
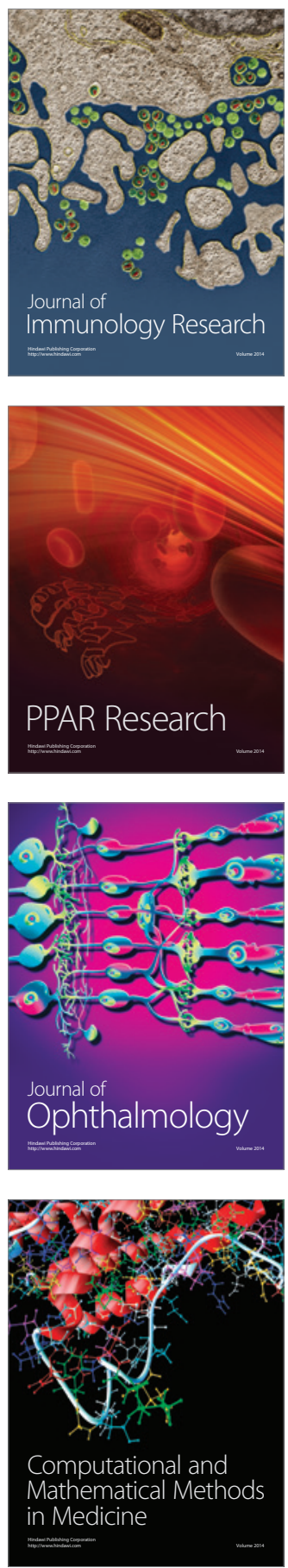

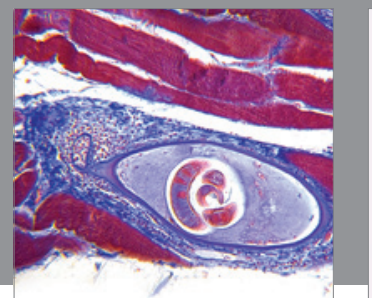

Gastroenterology

Research and Practice
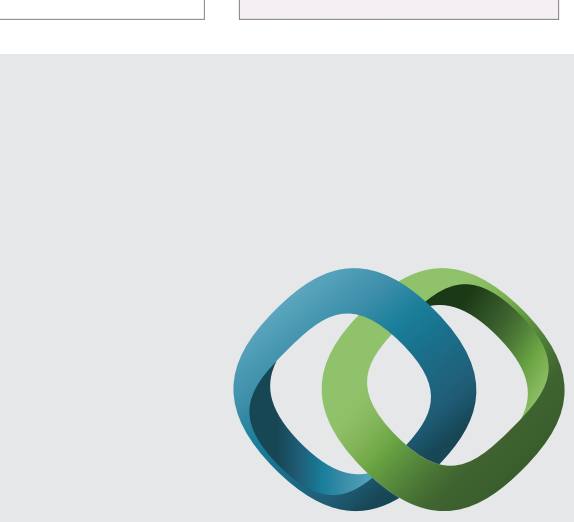

\section{Hindawi}

Submit your manuscripts at

http://www.hindawi.com
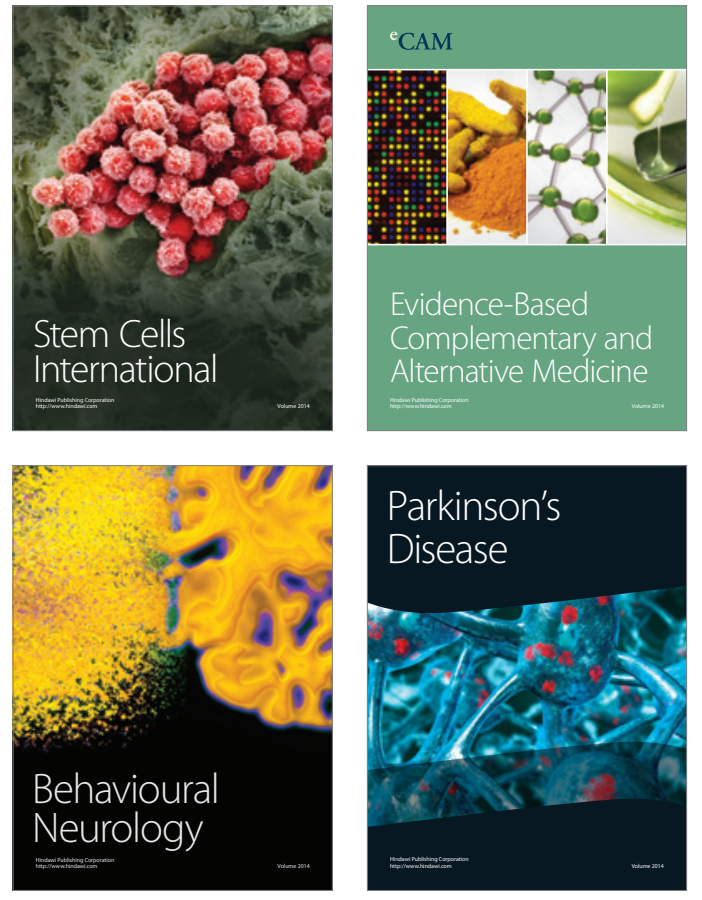
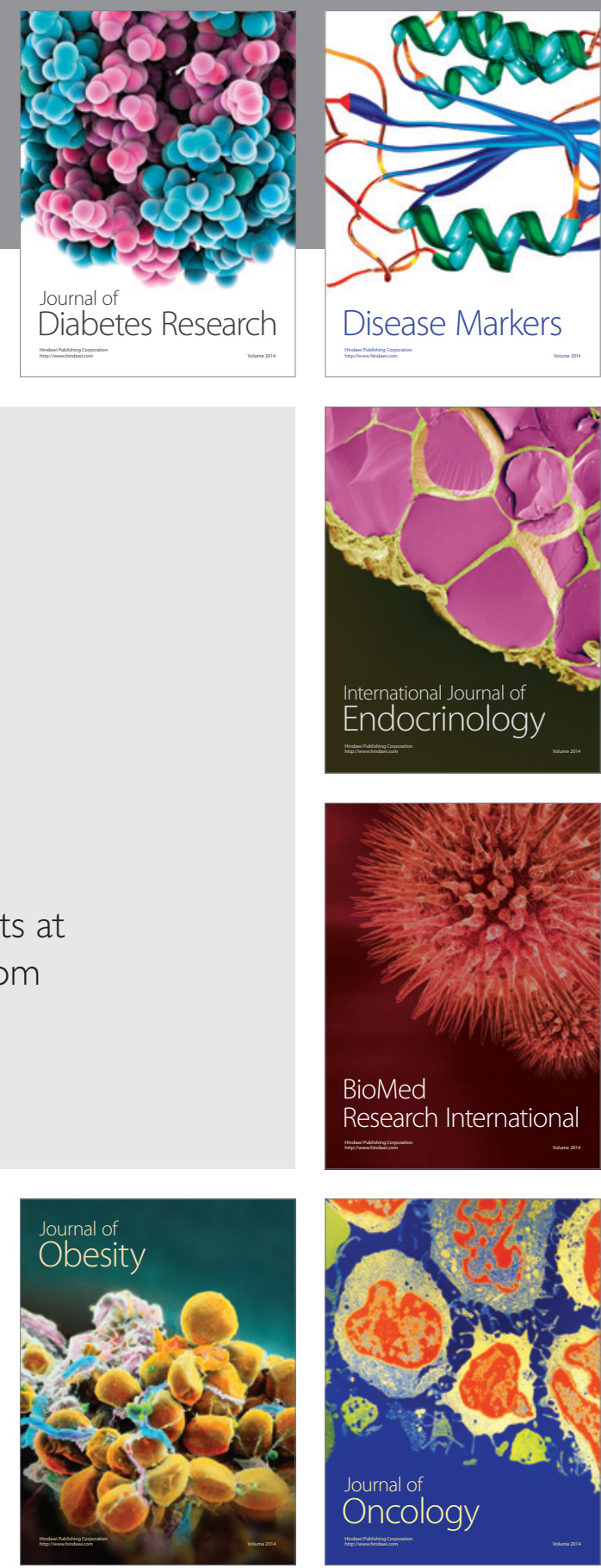

Disease Markers
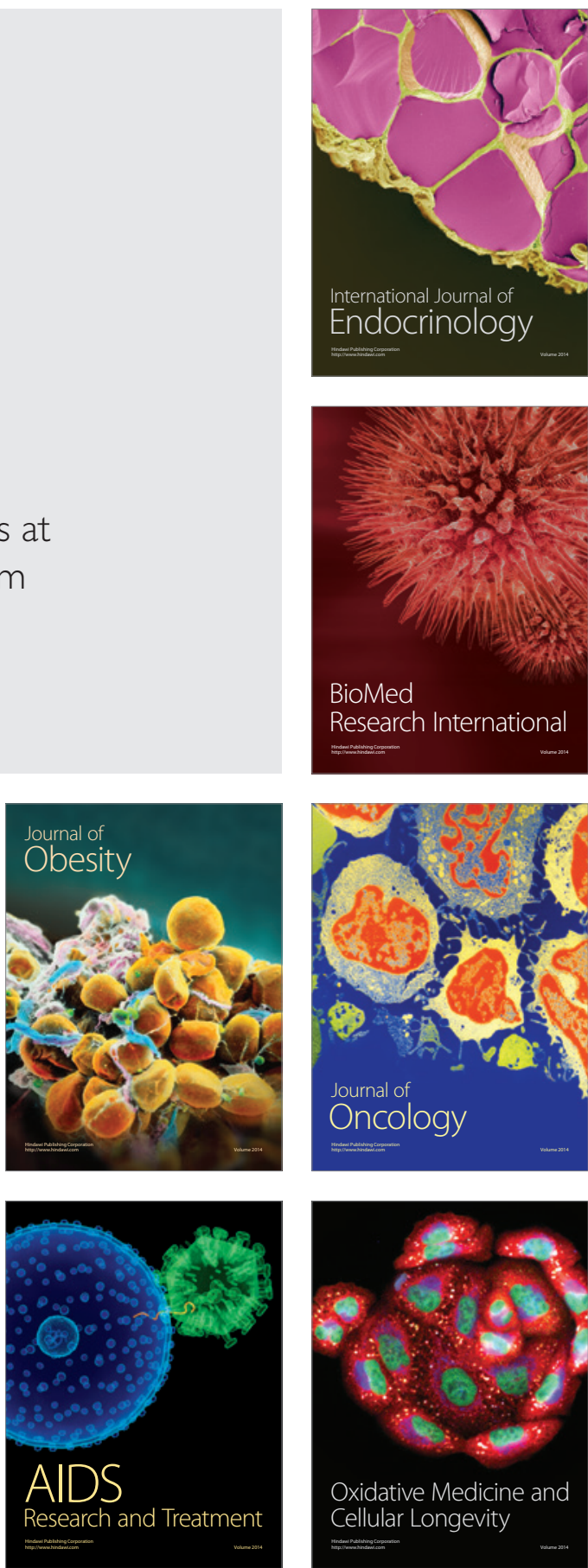